\title{
Análisis comparativo de cinco métodos de procesamiento para calcular el área de lagunas pampeanas a partir de imágenes satelitales Landsat
}

\author{
María Laura Maestri ${ }^{1,2,3 *}$, Florencia Castets ${ }^{1,2}$, Martín Bayala ${ }^{2,3,4}$ y Graciela Canziani ${ }^{1,2}$
}

'Instituto Multidisciplinario sobre Ecosistemas y Desarrollo Sustentable, Universidad Nacional del Centro de la Provincia de Buenos Aires, Paraje Arroyo Seco, (7000) Tandil, Buenos Aires,

Argentina. ${ }^{2}$ Comisión de Investigaciones Científicas de la Provincia de Buenos Aires, La

Plata, Buenos Aires, Argentina. ${ }^{3}$ Consejo Nacional de Investigaciones Científicas y Técnicas, Argentina. ${ }^{4}$ Instituto de Hidrología de Llanuras "Dr. Eduardo Jorge Usunoff", Universidad Nacional del Centro de la Provincia de Buenos Aires, Paraje Arroyo Seco, (7000) Tandil, Buenos Aires,

Argentina. * Corresponding author.

E-mail: mlmaestri@exa.unicen.edu.ar

RESUMEN. Las lagunas pampeanas evidencian una dinámica muy cambiante (estacional, anual o cíclicamente) en diversos aspectos: profundidad, superficie libre, turbidez, concentración de nutrientes, de clorofila, de sólidos en suspensión, conductividad, etc. Trabajos previos señalan una relación inversa entre el volumen de agua y los valores de indicadores de estado trófico, vinculando precipitaciones y vientos con el grado de eutrofia de estas lagunas. La comprensión de las interrelaciones complejas entre procesos biológicos y geofísicos se dificulta porque éstos no son lineales y presentan retardos que aún no están cabalmente identificados ni cuantificados, con posibles retroalimentaciones entre dichos procesos. La comprensión de la dinámica de la interacción entre clima y ecosistemas lénticos es fundamental para poder conservarlos, utilizando sus recursos y garantizando sus servicios. Un primer paso esencial para relacionar la evolución de las lagunas con las precipitaciones consiste en contar con algoritmos confiables para el procesamiento de imágenes satelitales. Presentamos aquí un análisis comparativo de cinco métodos computacionales para determinar la superficie de agua libre de la laguna La Barrancosa a partir de la aplicación de los índices NDWI (Índice de Diferencia Normalizada de Agua) y MNDWI (Índice de Diferencia Normalizada de Agua Modificado) y métodos supervisados (Máxima Verosimilitud) y no supervisados (ISODATA y K-means) de clasificación en imágenes Landsat (4TM, 5TM y 7ETM+) a lo largo del período 1984-2004. Esto permitió evaluar la capacidad de cada uno para seguir la dinámica del área superficial e identificar el método que insume menos tiempo computacional y minimiza posibilidades de error en la implementación.

Palabras clave: Clasificación de imágenes, laguna La Barrancosa, lagunas pampeanas, variabilidad.

ABSTRACT. Pampean shallow lakes exhibit very variable dynamics (seasonally, annually or cyclically) in various aspects: depth, free surface, turbidity, concentration of nutrients and Chl-a, suspended solids, conductivity, among others. Preliminary works indicate an inverse relationship between the volume of water and the values of trophic status indicators, linking rainfall and winds with the eutrophy degree of these lakes. The knowledge of the complex relationships between biological and geophysical processes is difficult because they are nonlinear and exhibit delays that are not yet fully identified nor quantified, with possible feedback between these processes. The understanding of the interaction dynamics between climate and lentic ecosystems is fundamental for their conservation, for resource utilization, and for services preserving. A first essential step to 
relate the evolution of the shallow lakes to precipitations is to achieve on reliable algorithms for the processing of satellite images. We introduce here a comparative analysis of five computational methods for estimating the area of free water surface of the La Barrancosa shallow lake in Landsat images (4TM, 5TM and 7ETM +) throughout the period 1984-2004, based on the application of NDWI (Normalized Water Difference Index) and MNDWI (Modified Normalized Water Difference Index) indices, as well as supervised (Maximum Likelihood) and unsupervised (ISODATA and K-means) classification methods. The performed analysis allowed us to evaluate the ability of each one to follow the dynamics of the surface area and to identify the method that requires less computational time and minimizes the possibility of error in the implementation.

Keywords: Image classification, La Barrancosa shallow lake, pampean shallow lakes, variability.

\section{INTRODUCCIÓN}

Las lagunas pampeanas se caracterizan por su numerosidad, -más de cien mil en la provincia de Buenos Aires-, por ser ambientes polimícticos, poco profundos y con variaciones frecuentes en su nivel hidrométrico y su superficie libre en función de las precipitaciones regionales y del balance hídrico subterráneo (Dangavs, 2005; Ringuelet, 1962). Las lagunas evidencian una dinámica muy cambiante, -sea estacionalmente, de año a año, o cíclicamente-, en las variables físicas, químicas y biológicas que condicionan sus características (Canziani et al., 2008; Cepeda et al., 2010; Kosten et al., 2012; Scheffer, 2004). En efecto, el estado trófico de las lagunas pampeanas, naturalmente eutrófico a hipereutrófico, se ve potenciado por el aporte de nutrientes provenientes de las actividades rurales y de vuelcos cloacales con o sin tratamiento (Grosman, 2008). Pero además, las lagunas están afectadas por inundaciones y sequías que afectan el volumen de agua y por la acción del viento que resuspende sedimentos, generando variaciones en la concentración de sales, turbidez y reducción de oxígeno (Grosman, 2008; Moss et al., 2003, 2011; Quirós et al., 2002). En consecuencia, los cambios observados en el estado trófico están relacionados con las variables climáticas a la vez que con la dinámica propia de cada laguna. El elevado número de lagunas en la extensa región pampeana hace que las comunidades locales no perciban la necesidad de cuidarlas, poniendo en riesgo la biodiversidad $y$, en consecuencia, la calidad de los servicios que prestan en el ecosistema y a las poblaciones. Con la observación adecuada de la dinámica de las lagunas pampeanas es posible identificar el impacto de las actividades humanas y de la variabilidad climática en su funcionamiento y en la calidad de los servicios ecosistémicos (Grosman et al., 2007a, b). El conocimiento de las respuestas a distintos factores ambientales posibilita diseñar formas de utilizar el ecosistema conservándolo, es decir desarrollar actividades y usos que sean eficientes económicamente, equitativos socialmente y sostenibles ambientalmente (Grosman, 2008).

La profusión de lagunas pampeanas distribuidas en una región tan vasta dificulta sensiblemente su monitoreo a campo, pero es posible obtener información con adecuada frecuencia a través de sensores remotos. En las últimas décadas, el procesamiento de imágenes satelitales se ha convertido en una herramienta esencial para monitorear los cuerpos de agua, proveyendo información vital para las sociedades humanas (Feyisa et al., 2014; Rokni et al., 2014; Zhang et al., 2016).

Con el propósito de identificar la relación entre el estado trófico de la laguna y las variables climáticas, surge la necesidad de determinar las variaciones en el área de la superficie libre de cada laguna. Para ello es fundamental contar con un método que permita un cálculo confiable del área a partir de una imagen satelital que, en este caso, es provista por la Misión Landsat. En este trabajo, se realizó la evaluación de cinco diferentes algoritmos de clasificación supervisada, no supervisada e índices espectrales para estimar la superficie libre de agua y evaluar su precisión con respecto a datos in situ de la laguna La Barrancosa. 


\section{MATERIALES Y MÉTODOS Sitio del estudio}

La laguna La Barrancosa $\left(37^{\circ} 20^{\prime} 00^{\prime \prime} \mathrm{S}, 60^{\circ} 06^{\prime}\right.$ 39" O) pertenece al partido de Benito Juárez, provincia de Buenos Aires, Argentina. Se encuentra ubicada a $47 \mathrm{~km}$ de la localidad de Olavarría, a 53 km de Benito Juárez y a 87 km de Tandil (Figura 1). Esta laguna responde a un régimen natural y no se encuentra sometida a manejo. El entorno muestra actividad agrícola y ganadera.

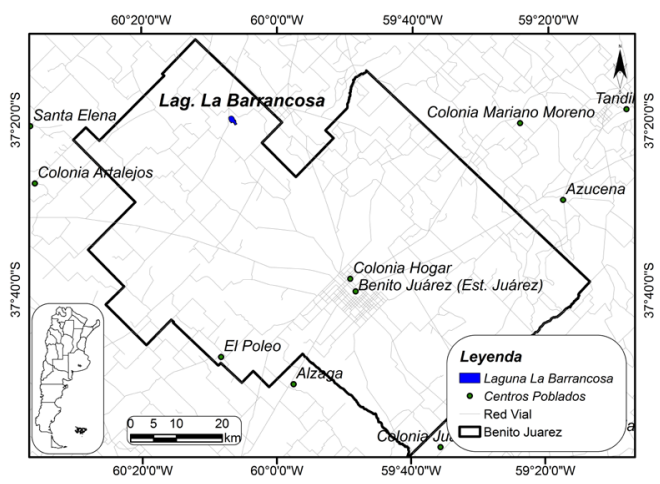

Figura 1. Área de estudio y localización de la laguna La Barrancosa.

Figure 1. Study area and location of the La Barrancosa shallow lake.

\section{Obtención y procesamiento de las imágenes satelitales}

Para analizar la dinámica de la superficie libre del cuerpo de agua se utilizó una serie de imágenes Landsat (Landsat 4 TM, Landsat 5 TM, Landsat 7 ETM+, path-row 225-86 y 22686) correspondientes al período 1984-2004. Se seleccionaron dos imágenes por año, una de verano y otra de invierno. Las mismas fueron adquiridas a través del repositorio web ESPA perteneciente al Servicio Geológico de Estados Unidos (USGS-ESPA (https://espa.cr.usgs.gov/)). Las imágenes de los sensores TM y ETM+ son provistas en nivel de procesamiento Level 2 , imagen geométrica y atmosféricamente corregida a nivel de superficie (Figura 2). Dicho producto es el resultado de la corrección del dato a nivel de tope de la atmósfera de efectos atmosféricos mediante la simulación a partir del modelo de transferencia radiativa Land Surface Reflectance Code (LaSRC-6S) (Vermote et al., 2016).

\section{Descripción de los métodos}

Para el cálculo de la superficie libre de las lagunas se utilizaron tres métodos de clasificación (K-means, ISODATA y Máxima Verosimilitud) y dos índices espectrales (NDWI y MNDWI) (Figura 2).

Dentro de los algoritmos basados en píxeles que se utilizaron se diferencian los paramétricos o no supervisados como el K-means y el ISODATA (Iterative Self-Organizing Data Analysis Technique), ambos algoritmos agrupan objetos en clases (clusterización) en función de sus características espectrales (Chuvieco, 1995). El agrupamiento se realiza minimizando la suma de distancias entre cada objeto y el centroide de su clase o cluster. El usuario fija la cantidad de clases (k) que el algoritmo debe identificar, se establecen k centroides, y cada píxel es asignado a su centroide más cercano; luego se actualiza la posición del centroide de cada grupo y se itera hasta que los centroides quedan fijos. El algoritmo ISODATA tiene algunas mejoras con respecto a K-means dado que permite la división y fusión de clases (Jensen, 2005). Las clases se fusionan o se dividen si se cumplen ciertas condiciones respecto de umbrales definidos por el usuario. Para los clasificadores no supervisados K-means e ISODATA se utilizaron una cantidad máxima de 100 iteraciones, número de clases a discriminar de 5 , umbral de cambio (\%), máximo desvío estándar de 1 y error de distancia por clase de 5.

El método de clasificación paramétrico supervisado de Máxima Verosimilitud (Chuvieco, 1995) supone que los valores de reflectividad en cada una de las clases siguen una distribución de probabilidad normal multivariante. Se utiliza el vector de medias y la matriz de varianzascovarianzas para estimar la probabilidad de que un píxel dado pertenezca a cada una de las clases. El pixel se asignará a la clase cuya probabilidad de pertenencia sea mayor. Una vez terminada la asignación de píxeles a las distintas clases, el usuario establece umbrales de probabilidad para cada categoría, rechazando los píxeles con una probabilidad muy baja, evitando así que se clasifiquen píxeles en clases incorrectas. De esta manera, tendremos un número de píxeles sin clasificar, y en el caso de ser muy grande se debe retornar la fase 
de entrenamiento para deducir así nuevas categorías o perfeccionar las ya existentes. En todo caso el valor de probabilidad máximo puede utilizarse como indicador de la certeza en la clasificación. Este método se basa en la idea de, dada una muestra, hallar los valores de los parámetros que hacen que la probabilidad de obtener dicha muestra sea máxima. Para el clasificador de Máxima Verosimilitud se realizó la selección de muestras para entrenamiento a partir de píxeles contenidos en la región de interés con el propósito de condicionar el aprendizaje del algoritmo. Para el aprendizaje es necesario estimar una función de densidad de probabilidad del modelo estadístico de cada clase a priori y posteriori, que se realizó automáticamente con el software ENVI.

El índice NDWI (Índice de Diferencia Normalizada de Agua) propuesto por McFeeters, (1996) y el MNDWI (Índice de Diferencia Normalizada de Agua Modificado) por Xu (2006) se utilizaron para obtener una adecuada estimación del agua en superficie y el dosel de la vegetación a partir de la información espectral, están definidos respectivamente de la siguiente manera:

$$
\begin{aligned}
& N D W I=\frac{\text { Verde }-I R C}{\text { Verde }+I R C} \\
& M N D W I=\frac{\text { Verde }-I R M}{\text { Verde }+ \text { IRM }}
\end{aligned}
$$

donde, Verde representa el valor de la reflectancia en la banda correspondiente a la longitud de onda en el rango 450-520 nm, IRC el valor de la reflectancia entre las longitudes de onda 760-900 nm e IRM en el infrarrojo medio entre las longitudes de onda 1550-1750 nm. La relación entre bandas permite maximizar la reflectancia del agua al trabajar con longitudes de ondas en el verde, maximiza la reflectancia de la vegetación y minimiza la reflectancia de masas de agua gracias a la banda del infrarrojo cercano (IRC) y del infrarrojo medio (IRM). De esta manera, la presencia de agua se identifica en la imagen que se obtiene, con valor de cada píxel en un rango de valores entre - 1 y 1 debido a su definición matemática (Ec. 1 y 2). Los valores positivos del MNDWI indican presencia de agua, mientras que para el NDWI, se debe determinar en cada caso el umbral a partir del cual se registra presencia de agua lo cual implica la posibilidad de mayor error y tiempo de procesamiento.

\section{Métricas}

Las métricas estadísticas que fueron utilizadas en la evaluación de los métodos de procesamiento empleados están basadas en el análisis de errores. También, se identificaron las áreas máximas y mínimas por cada método, con el objeto de analizar si los métodos coincidían en la detección de estos eventos extremos.

Cabe notar que no hay un método directo a campo para calcular el área de la superficie libre de un cuerpo de agua y que en todos los casos se obtiene una estimación indirecta sea a través de una imagen satelital, sea a través de una aplicación de GPS que también depende de uno o varios satélites. En tal sentido, se tomó el valor promedio de los resultados de los cinco métodos para cada una de las fechas $i$, como estimación de referencia o verdad de campo, que se denominó $v r(i)$. A partir de éste se estimó la distancia respecto del valor de referencia vr como información de la posición de un dato respecto del conjunto de datos. Para cada método, se tomó para cada fecha $i$ la diferencia o error absoluto (EA) entre el área obtenida aplicando ese método (estimada) y el área de referencia $\operatorname{vr}(i)$.

$$
E A(i)=\text { área (i) }-v r(i)
$$

Estas diferencias permiten calcular la magnitud del error relativo $(E R)$ respecto del valor de referencia $v r(i)$ a partir de la siguiente expresión:

$$
E R(i)=\frac{\mid \text { área }(i)-v r(i) \mid}{\operatorname{vr}(i)}
$$

El máximo error relativo alcanzado permite obtener un indicador de la precisión del método. Sin embargo, como sólo se obtiene en referencia a una evaluación puntual, esta norma no permite apreciar cuán buenos o malos fueron los resultados obtenidos para el conjunto de las observaciones. Para tener una medida global de la bondad de un método dado para toda la serie de imágenes utilizadas, 
se calculó el error global $(E G)$ como promedio de estos $E R$ obtenidos con este método sobre dicha serie. En otros términos, se calculó cuánto se apartan en promedio las áreas calculadas con este método del valor de referencia a lo largo de las $N$ fechas consideradas:

$$
E G=\frac{1}{N} \quad \sum_{i=1}^{N} E R(i)
$$

También fue considerado el error euclídeo $(E E)$ de los valores obtenidos con cada método respecto del promedio, dado que representa la acumulación de los errores a lo largo de la totalidad de fechas consideradas:

$$
E E=\sqrt{\sum_{i=1}^{N}(\operatorname{area}(i)-\operatorname{vr}(i))^{2}}
$$

donde, área (i) es el área calculada por el método considerado para la fecha $i$ y $v r(i)$ es el valor de referencia para la misma fecha $i$ (Figura 2).

Finalmente, para realizar un ensayo a campo, se procedió a recorrer a pie el perímetro de la laguna llevando un GPS Garmin e-trex 30x (precisión en terreno de $\pm 3,6 \mathrm{~m}$ ) con una aplicación que permite el cálculo del área comprendida en un recorrido cerrado. A partir de medidas in situ realizadas con dicho GPS para el día 08/11/2018 y la estimación de la superficie de la laguna obtenida de una imagen Landsat 8 OLI (02/10/2018) se realizó la evaluación puntual de los métodos propuestos.

\section{RESULTADOS Y DISCUSIÓN}

Los resultados obtenidos a partir de algoritmos de clasificación supervisados (Máxima Verosimilitud), no supervisados (ISODATA y K-means) e índices espectrales (NDWI y MNDWI), permitieron evaluar la capacidad de los mismos para seguir las dinámica del área de la laguna La Barrancosa para el período comprendido entre los años 1984 y 2004, posibilitando así evaluar su precisión y costo computacional.

Los cinco métodos reflejan una dinámica similar en la evolución del área de la laguna La Barrancosa; y la variación en la estimación del área por cada método y respecto del vr (Figura 3, promedio de los cinco resultados).

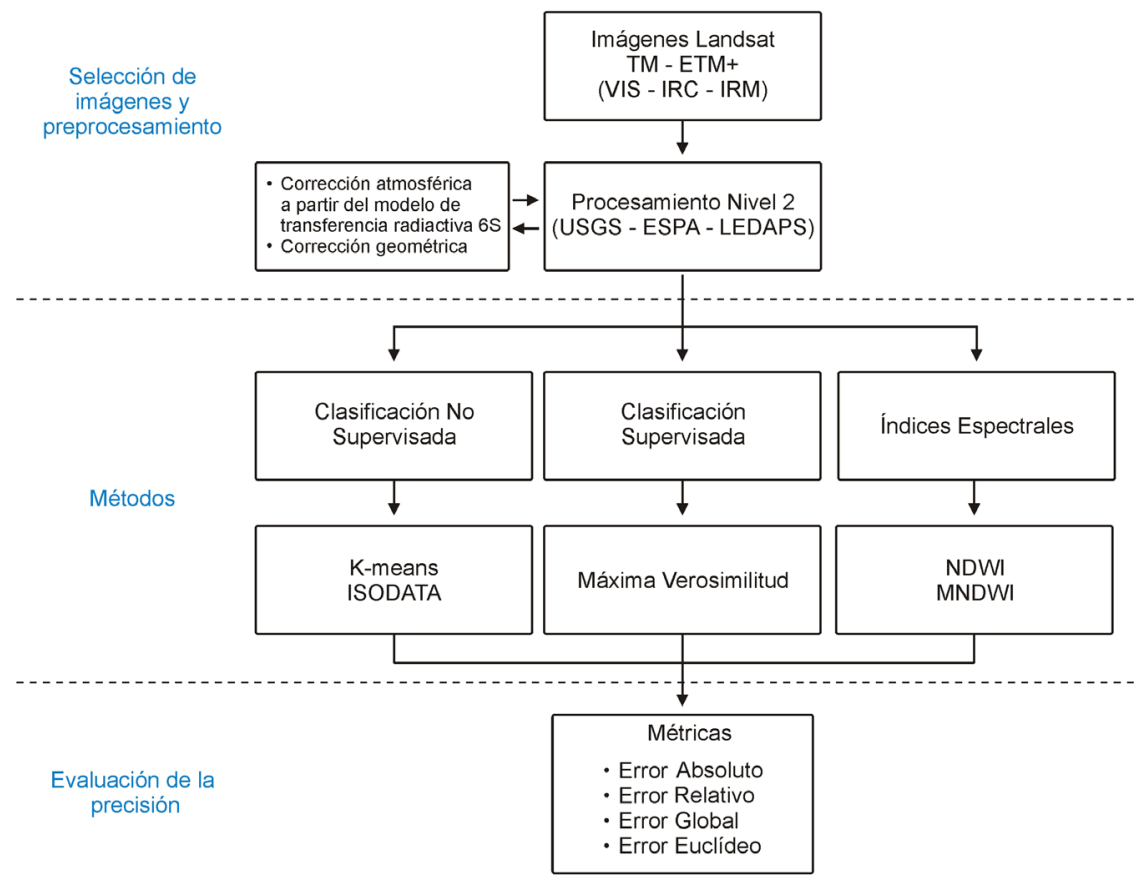

Figura 2. Flujograma de la metodología empleada. Figure 2. Flowchart of the employed methodology. 
Se usó la distancia respecto del valor de referencia $v r$ como información de la posición de un dato respecto del conjunto de datos. Este análisis permite tener una primera aproximación al comportamiento de los métodos en la determinación de área, pero no basta para determinar o inferir cuál método es más preciso. Tomando el valor de referencia como "verdad", se calcularon las diferencias o "errores" en las estimaciones del área de la laguna La Barrancosa obtenidas de cada método (Figura 4).

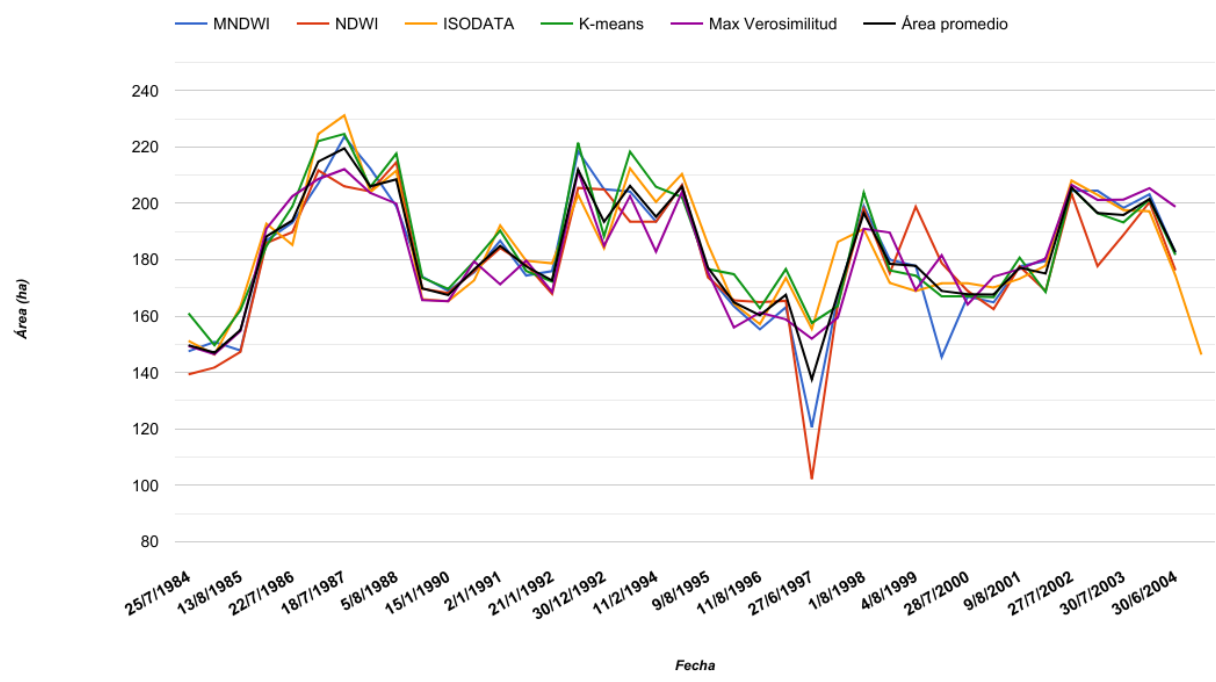

Figura 3. Variación del área de la laguna La Barrancosa para el período 1984-2004 y el valor de referencia (área promedio).

Figure 3. Area variation of the La Barrancosa shalow lake for the period 1984-2004 and the reference value (average area).
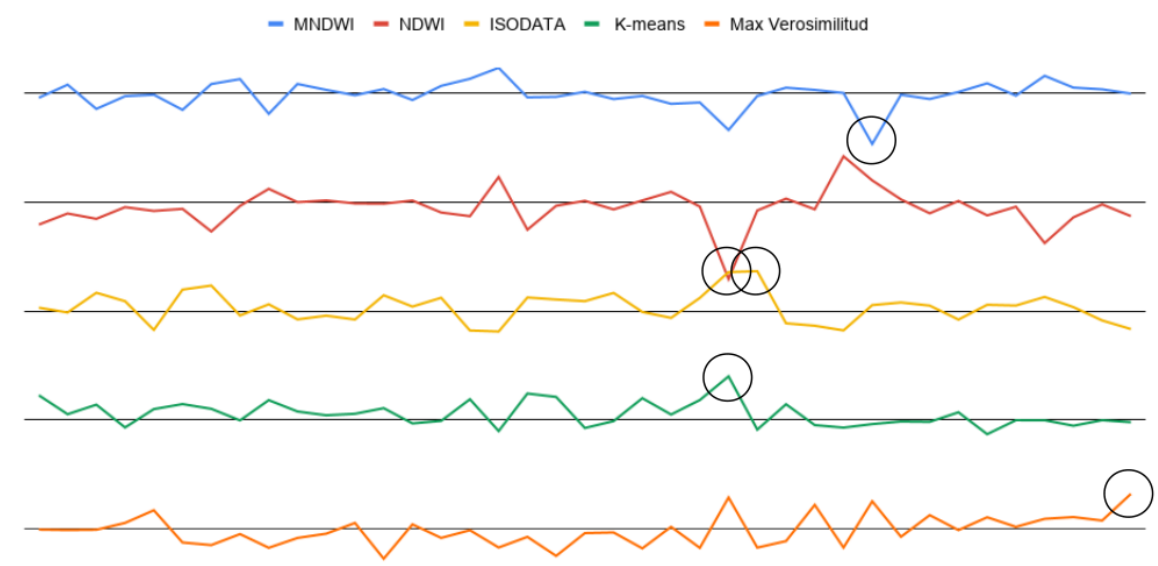

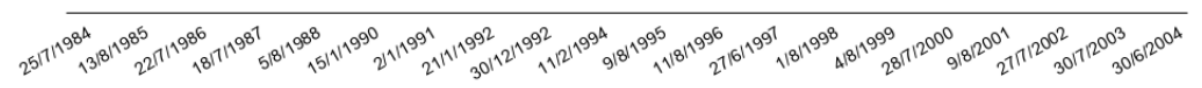

Figura 4. Gráficos de los errores $E A(i)$ del valor obtenido respecto al valor de referencia (vr) para cada uno de los métodos. El círculo señala el mayor $E A(i)$ observado.

Figure 4. Graphs of the $E A(i)$ errors between the calculated value and the reference value (vr) for each method. The circle shows the largest observed $E A(i)$. 
Con esta información se procedió a calcular los errores relativos $(E R)$ estimados para cada método en cada fecha, los errores relativos globales $(E G)$ de cada método sobre la serie de 40 imágenes, así como el error euclídeo (EE) acumulado por cada método sobre la serie de imágenes (Tabla 1).

Tabla 1. Errores de cada método respecto del valor de referencia $(v r)$

Table 1. Errors for each method with respect to the reference value (vr).

\begin{tabular}{|cccccc|}
\hline Método & MNDWI & NDWI & ISODATA & K-means & $\begin{array}{c}\text { Máxima } \\
\text { Verosimilitud }\end{array}$ \\
\hline EA [ha] & $\begin{array}{c}23,40 \\
(27 / 1 / 2000) *\end{array}$ & $\begin{array}{c}35,33 \\
(27 / 6 / 1997)\end{array}$ & $\begin{array}{c}18,25 \\
(28 / 1 / 1998)\end{array}$ & $\begin{array}{c}20,02 \\
(27 / 6 / 1997)\end{array}$ & $\begin{array}{c}16,09 \\
(30 / 6 / 2004)\end{array}$ \\
\hline ER [\%] & $\begin{array}{c}13,85 \\
(27 / 1 / 2000)\end{array}$ & $\begin{array}{c}25,68 \\
(27 / 6 / 1997)\end{array}$ & $\begin{array}{c}12,98 \\
(27 / 6 / 1997)\end{array}$ & $\begin{array}{c}14,55 \\
(27 / 6 / 1997)\end{array}$ & $\begin{array}{c}10,49 \\
(27 / 6 / 1997)\end{array}$ \\
\hline EG [\%] & 2,35 & 3,38 & 3,20 & 2,70 & 3,15 \\
EE [ha] & 38,88 & 56,45 & 43,73 & 39,75 & 44,39 \\
\hline *Entre paréntesis figura la fecha en la que se observó ese error. & \\
\hline
\end{tabular}

Debe destacarse que, si se consideran los errores máximos, tanto absoluto $(E A(i))$ como relativo $(E R(i))$, que indican puntualmente lo que ocurre en una fecha $i$, Máxima Verosimilitud aparenta ser el más preciso. Sin embargo, esto no muestra la eficiencia real de este método dado que cuando se evalúa el rendimiento sobre la serie completa de imágenes este método queda relegado. Los valores de las dos últimas filas de la Tabla 1 ponen en relieve la bondad de MNDWI entre los índices y K-means entre los métodos de clasificación, mientras que NDWI, ISODATA y Máxima Verosimilitud presentan a su vez los mayores valores acumulados de error. La Tabla 2 muestra las diferencias y el correspondiente porcentaje de error obtenidos al tomar como valor de referencia o valor de campo el área calculada por la aplicación del GPS Garmin e-trex 30 (186,02 ha) en el terreno. Se observa que el algoritmo de Máxima Verosimilitud presentó la mayor diferencia de área (> 20 ha) mientras que, en concordancia con los resultados obtenidos en la Tabla 1, para el índice MNDWI se observó el porcentaje de error más bajo ( $<0,01$ ha). La Figura 5 muestra en verde el trazado registrado por el aparato GPS superpuesto a la imagen Landsat 8 de la fecha 02/10/2018 procesada utilizando el índice MNDWI.
Tabla 2. Cálculo del área a partir de los métodos analizados y su diferencia con datos de campo

Table 2. Area computed using the analyzed methods and the difference with respect to terrain data

\begin{tabular}{cccc}
\hline \multicolumn{1}{c}{ Método } & Área [ha] & Diferencia [ha] & Error (\%) \\
\hline GPS & 186,02 & 0 & 0 \\
MNDWI & 186,03 & 0,01 & 0,01 \\
NDWI & 179,91 & 6,11 & 3,28 \\
ISODATA & 179,37 & 6,65 & 3,57 \\
K-means & 178,29 & 7,73 & 4,15 \\
$\begin{array}{c}\text { Máxima } \\
\text { Verosimilitud }\end{array}$ & 165,06 & 20,96 & 11,27 \\
\hline
\end{tabular}

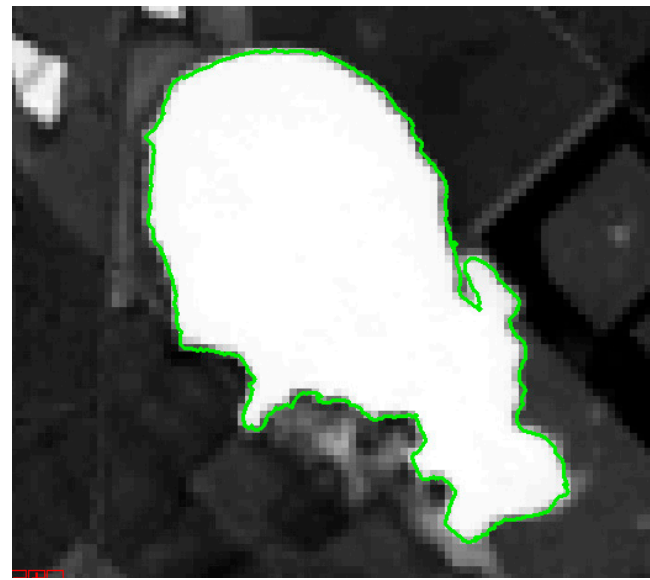

Figura 5. Imagen L8_226-086_2018-10-02 obtenida con MNDWI. La línea verde indica el recorrido realizado a pie según el GPS.

Figure 5. Image L8_226-086_2018-10-02 resulting from MNDWI. The green line shows the walking tour according to GPS.

Respecto de la capacidad de cada uno de los métodos de detectar áreas extremas, cabe mencionar que el área mínima para los índices MNDWI y NDWI se obtuvo en la fecha 27/06/1997 mientras que para los métodos ISODATA, K-means y Máxima Verosimilitud se registró en la fecha 01/01/1985. Con respecto al área máxima, todos los métodos coinciden en la fecha 18/07/1987 con la excepción del índice NDWI que logra su área máxima en la fecha 05/8/1988 (Tabla 3).

Clark et al. (2001) señalaron la importancia de desarrollar la capacidad de predecir la evolución de los ecosistemas y los servicios ecosistémicos para definir políticas de preservación y 
Tabla 3. Área [ha] mínima, máxima e imagen de los diferentes métodos aplicados.

Table 3. Minimum and maximum area [ha] and imagery of the different applied methods.

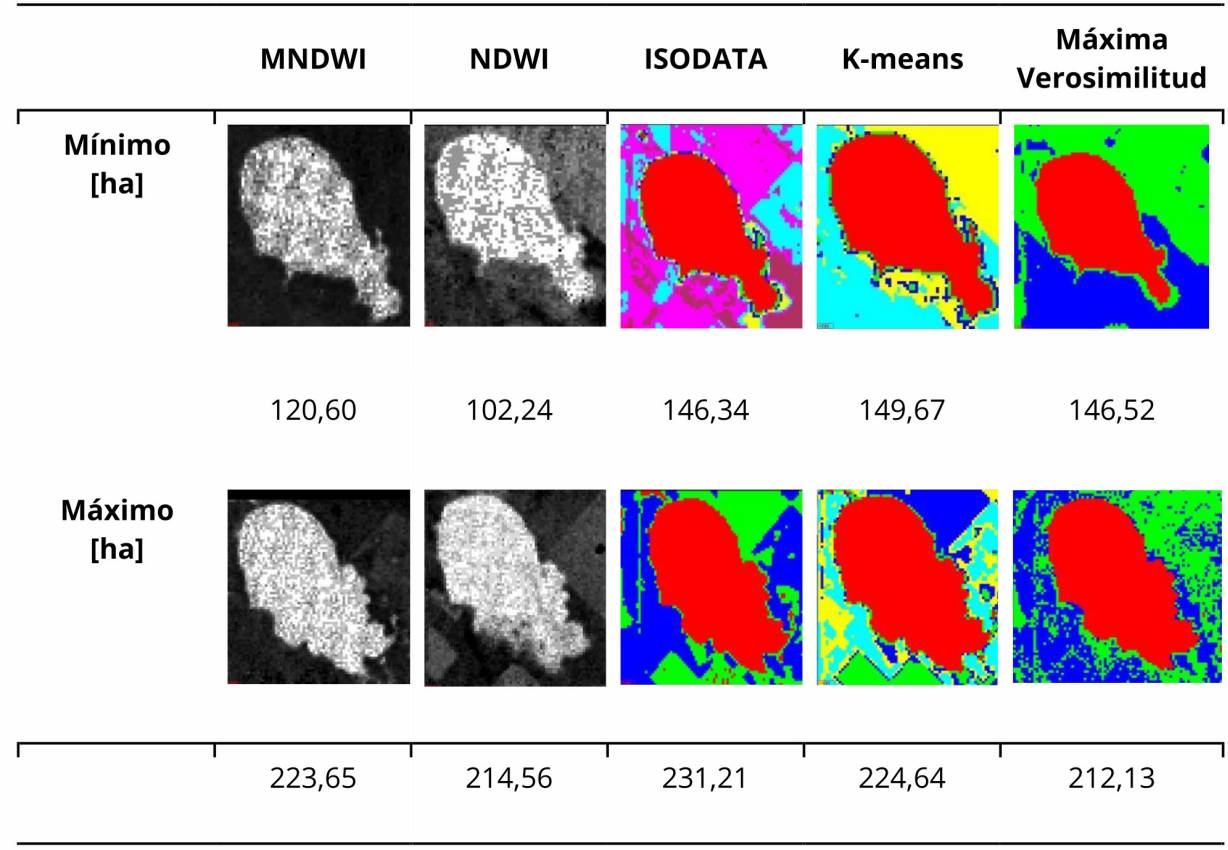

manejo. Entre los factores que sin lugar a dudas impactan la dinámica, estado trófico y servicios de los humedales y lagunas, y que es imprescindible tener en cuenta, figuran las actividades antrópicas y el clima (Canziani et al., 2008; Cepeda et al., 2010; Diovisalvi et al., 2014; Quirós et al., 2006 ).

El desarrollo de modelos estadísticos y/o matemáticos, así como de herramientas computacionales, de observación y de monitoreo con fines de diagnóstico y perfeccionamiento de la capacidad predictiva, es de particular importancia para fortalecer el conocimiento de la dinámica de los ecosistemas lénticos pampeanos y analizar su estructura y funcionamiento. En este sentido, y considerando la extensión de la región pampeana, el procesamiento de imágenes satelitales se vuelve una herramienta esencial para monitorear los cuerpos de agua, proveyendo información útil a estos fines. Desde luego, los métodos de procesamiento deben ser los adecuados a las características del ecosistema y confiables en cuanto a la precisión de sus resultados.

Los cinco métodos analizados aquí responden a diferentes conceptos y muestran distintos grados de complejidad y de necesidad de información para obtener resultados confiables. Los índices MNDWI y NDWI se caracterizan por la sencillez del algoritmo. Los métodos de clasificación no supervisados ISODATA y K-means exigen la definición de parámetros, de cuyo ajuste depende la calidad del resultado. Es posible mejorar su rendimiento con pruebas respecto el número de iteraciones, definición de umbrales o números de pixeles para maximizar dicho ajuste. El método de clasificación supervisada de Máxima Verosimilitud requiere de información de campo con cierto detalle que no necesariamente puede estar disponible.

Al analizar los datos de la Tabla 1 puede deducirse que el índice MNDWI y el clasificador $\mathrm{K}$-means entregan resultados con errores equivalentes, calculados desde distintas perspectivas, en ambos casos menores a los de los otros tres métodos. Sin embargo, en este estudio los resultados obtenidos han permitido identificar al índice MNDWI no sólo como el de menor error sino también como el método más sencillo y rápido desde el punto de vista computacional para calcular el área de la laguna La Barrancosa. En consecuencia, MNDWI resulta un método computacional práctico y confiable para determinar la presencia o ausencia de 
superficies de agua libre en la región pampeana en imágenes Landsat.

\section{AGRADECIMIENTOS}

Maestri M. L. posee una beca doctoral del Consejo Nacional de Investigaciones Científicas y Técnicas de Argentina (CONICET). Castets F. y Bayala M. son Personal de Apoyo de la Comisión de Investigaciones Científicas de la Provincia de Buenos Aires (CICPBA).

\section{REFERENCIAS}

Canziani, G. A., Ferrati, R., Dukatz, F. y Marinelli, C. (2008). Artificial neural networks and remote sensing in the analysis of the dynamics of highly variable shallow lakes. Mathematical Biosciences and Engineering, Special Issue honouring Thomas G. Hallam, 5 (4): 691 -711.

Cepeda, R., Colasurdo, V., Díaz, O., Ferrati, R., Grosman, F., Marinelli, C., Sanzano, P. y Canziani, G. (2010). Elaboración de un índice de estado trófico adaptado a categorizar lagunas pampeanas. En: Varni, M., Entraigas, I. y L. Vives (Eds.) Libro Actas I Congreso Internacional de Hidrología de Llanuras, Tomo II, Hacia la gestión integral de los recursos hídricos en zonas de Ilanura. (pp. 385-392).

Chuvieco Salinero, E. (1995). Fundamentos de teledetección espacial. Madrid: Rialp.

Clark, J. S., Carpenter, S. R., Barber, M., Collins, S., Dobson, A., Foley, J. A., Lodge, D. M., Pascual, M., Pielke Jr, R., Pizer, W., Pringle, C., Reid, W. V., Rosel, K. A., Sala, O., Schlesinger, W. H., Wall, D. H. \& Wear, D. (2001). Ecological Forecasts: An Emerging Imperative. Science, 293: 657-660.

Dangavs, N. V. (2005). Los ambientes acuáticos de la provincia de Buenos Aires. En: Barrio, R. E., Etcheverry, R. O., Caballé, M. F., \& Llambías, E. J. (Eds.). Geología y recursos minerales de la Provincia de Buenos Aires. (219-236). XVI Congreso Geológico Argentino, Relatorio 13: La Plata.

Diovisalvi, N., Bohn, V. Y., Piccolo, M. C., Perillo, G. M., Baigún, C. \& Zagarese, H. E. (2014). Shallow lakes from the Central Plains of Argentina: an overview and worldwide comparative analysis of their basic limnological features. Hydrobiologia,752 (1): 5-20.

Feyisa, G. L.; Meilby, H.; Fensholt, R, Proud, S. R. (2014). Automated Water Extraction Index: A new technique for surface water mapping using Landsat imagery. Remote Sensing of Environment, 140: 23-35

Grosman, F., Sanzano, P., Díaz, O., Colasurdo, V., Ferrati, R., Dukatz, F. y Canziani, G. (2007a). Lagunas de la región pampeana de Argentina: estructura y funcionamiento a partir de aspectos sociales, económicos, culturales y naturales. En: Feyen, J., Aguirre L.F., Moraes M. R. (Eds.). Congreso Internacional sobre Desarrollo, Medio Ambiente y Recursos Naturales: Sostenibilidad a Múltiples Niveles y Escalas. Junio de 2007. Cochabamba, Bolivia. (pp. 1130-1137).

Grosman, F., Sanzano, P. \& Rudzik, G. (2007b). Incidence of local and global changes on the dynamics of pampean shallow lakes: the case of Blanca Grande. En: Scarpati, O. \& Jones, J.A.A. (Eds.). Environmental Change and Rational Water Use (pp. 294-301). Buenos Aires: Orientación Gráfica Editora.

Grosman, F. (2008). Espejos en la llanura: Nuestras lagunas de la región pampeana. Buenos Aires:

Editorial Universidad Nacional del Centro de la Provincia de Buenos Aires.

Jensen, J. R. (2005). Introductory digital image processing: a remote sensing perspective. United States of America: Prentice Hall series in geographic information science.

Kosten, S., Vernooij, M., Van Nes, E. H., Sagrario, M. A., Clevers, J. G. \& Scheffer, M. (2012). Bimodal transparency as an indicator for alternative states in South American lakes. Freshwater Biology, 57 (6): 1191-1201.

Mc Feeters, S. K. (1996). The use of the Normalized Difference Water Index (NDWI) in the delineation of open water features. International Journal of Remote Sensing, 17 (7): 1425-1432.

Moss, B., Mckee, D., Atkinson, D., Collings, S. E., Eaton, J. W., Gill, A. B., Harvey, I., Hatton, K., Heyes, T. \& Wilson, D. (2003). How important is climate? Effects of warming, nutrient addition and fish on phytoplankton in shallow lake microcosms. Journal of Applied Ecology, 40: 782-792. 
Moss, B., Kosten, S., Meerhoff, M., Battarbee, R. W., Jeppesen, E., Mazzeo, N., Havens K., Lacerot, G., Liu, Z., De Meester, L., Paerl, H. \& Scheffer, M. (2011). Allied attack: climate change and eutrophication. Inland Waters, 1: 101-105.

Quirós, R, Rennella, A., Boveri, M., Rosso, J. J. y Sosnovsky, A. (2002). Factores que afectan la estructura y el funcionamiento de las lagunas pampeanas. Ecología Austral, 12: 175-185.

Quirós, R., Boveri, M. B., Petracchi, C. A., Rennella, A. M., Rosso, J. J., Sosnovsky, A., \& Von Bernard, H. T., (2006). Los efectos de la agriculturización del humedal pampeano sobre la eutrofización de sus lagunas. En: Tundisi, J. G., MatsumuraTundisi, T. \& Sidagis-Galli, C. (Eds.). Eutrofização na América do Sul: Causas, conseqüências e tecnologias de gestão (pp. 1 - 16). São Carlos, Brasil: Rede EUTROSUL, PROSUL, Instituto Internacional de Ecologia.

Ringuelet, R. A. (1962). Rasgos principales de las lagunas pampeanas con criterio bioecológico. Anales CIC, III: 315-339.
Rokni, K., Ahmad, A., Selamat, A. \& Hazini, S. (2014). Water feature extraction and change detection using multitemporal Landsat imagery. Remote Sensing, 6 (5): 4173-4189.

Scheffer, M. (2004). Ecology of shallow lakes. Springer: Dordrecht.

Vermote, E., Justice, C., Claverie, M. \& Franch, B. (2016). Preliminary analysis of the performance of the Landsat 8/OLI land surface reflectance product. Remote Sensing of Environment, 185: 46-56.

Xu, H. (2006). Modification of normalised difference water index (NDWI) to enhance open water features in remotely sensed imagery. International Journal of Remote Sensing, 27(14): 3025-3033.

Zhang, F., Tiyip, T., Johnson, V. C., Wang, J. y Nurmemet, I. (2016). Improved water extraction using Landsat TM/ETM+ images in Ebinur Lake, Xinjiang, China. Remote Sensing Applications: Society and Environment, 4: 109-118. 